\title{
Long-Term Reproducibility of Coronary Flow Velocity Measurements in Patients With Coronary Artery Disease
}

\author{
Carlo Di Mario, MD, PhD, Robert Gil, MD, and Patrick W. Serruys, MD, PhD
}

$\mathbf{N}$ ew developments in transducer technology and signal analysis have increased the applicability of intracoronary Doppler ultrasonography for the measurement of coronary blood flow velocity. Intracoronary Doppler guide wires are used to estimate the severity of intermediate lesions, ${ }^{1}$ to define the functional results of coronary interventions, ${ }^{2}$ and to study coronary flow changes after pharmacological treatment. ${ }^{3}$ A prerequisite for these clinical and research applications is the validation of the long-term reproducibility of flow velocity measurements. This study assesses the long-term changes of baseline and hyperemic flow velocity and coronary flow reserve in 31 patients with coronary artery disease.

$$
\text { -. }
$$

Thirty-one patients with stable angina and single-vessel disease (age $58 \pm 9$ years, 23 men and 8 women) undergoing percutaneous revascularization participated in this study. None of the patients had anemia, hyperthyroidism, or diabetes mellitus. Systemic hypertension was present in 6 of 31 patients (19\%). All vasoactive medication, with the exception of short-acting nitrates, was interrupted 48 hours before the study. A 0.018 -inch or 0.014-inch diameter Doppler guide wire (FloWire, Cardiometrics, Mountain View, California) was advanced into the mid-segment of an artery that was without diameter reductions greater than $30 \%$, was not originating collaterals for the occluded vessel, and was without wall contraction abnormalities in its territory of distribution. The left anterior descending coronary artery was studied in 6 patients, the left circumflex in 13 patients, and the right coronary artery in 12 patients. Seven- to 9 Fr guiding catheters were used for the left coronary artery, and 7Fr diagnostic catheters for the right coronary artery. Flow velocity

\footnotetext{
From the Intracoronary Imaging Labora toy and the Cardiac Catheterization Laboratory, Division of Cardiology. Tho raxcenter, Erasmus University, Rotterdam, The Netherlands. Dr. R. Gil is the recip ient of the Training Fellowship 1994 of the European Society of Cardiology. Presented in part at the 67 th Scientific Sessions of the American Heart Association, Dallas, Texas, November 1994. Dr. Serruys' address is: Cardiac Calheterization Laboratory, Division of Cardiology, Thoraxcenter, Bd 408, Erasmus University, P.O. Box 1738, 3015 GD Rotterdam, The Netherlands. Manuscript received February 2, 1995; revised manuscript received and accepted Feb ruary 16,1995
}

was recorded (FloMap, Cardiometrics, Mountain View, California) at the peak effect of an intracoronary bolus of 12 (left coronary artery) or $8 \mathrm{mg}$ (right coronary artery) of papaverine, after withdrawal of the guiding catheter if signs of obstruction to flow were present. Five minutes after the injection of papaverine, allowing the restoration of basal conditions, a new flow velocity and a cineangiogram were recorded (Figure 1). During measurement of hyperemic and baseline velocity, heart rate and aortic blood pressure were simultaneously recorded and measured. After a follow-up period of 4 to 7 months (mean 5.8 months), hyperemic and baseline coronary flow velocity were recorded in the same position and by using the same material and protocol.

The Doppler system that was used automatically calculates on-line a temporal average (mean of two beats) of the peak velocity, detected after spectral analysis of the Doppler signal. ${ }^{4}$ Manual retracing and calculation with an off-line system was required for $16 \%$ of the measurements because of partial or complete failure of the detection algorithm. Coronary flow reserve was calculated as the ratio between mean (time-averaged peak) hyperemic velocity and baseline velocity. The vessel diameter at the site of the Doppler sample volume was measured off-line using a cine-film system (CAAS 2, Pie Medical Data, Maastricht, The Netherlands) based on the computer-assisted application of an automatic edgedetection algorithm. The angiographic catheter was used

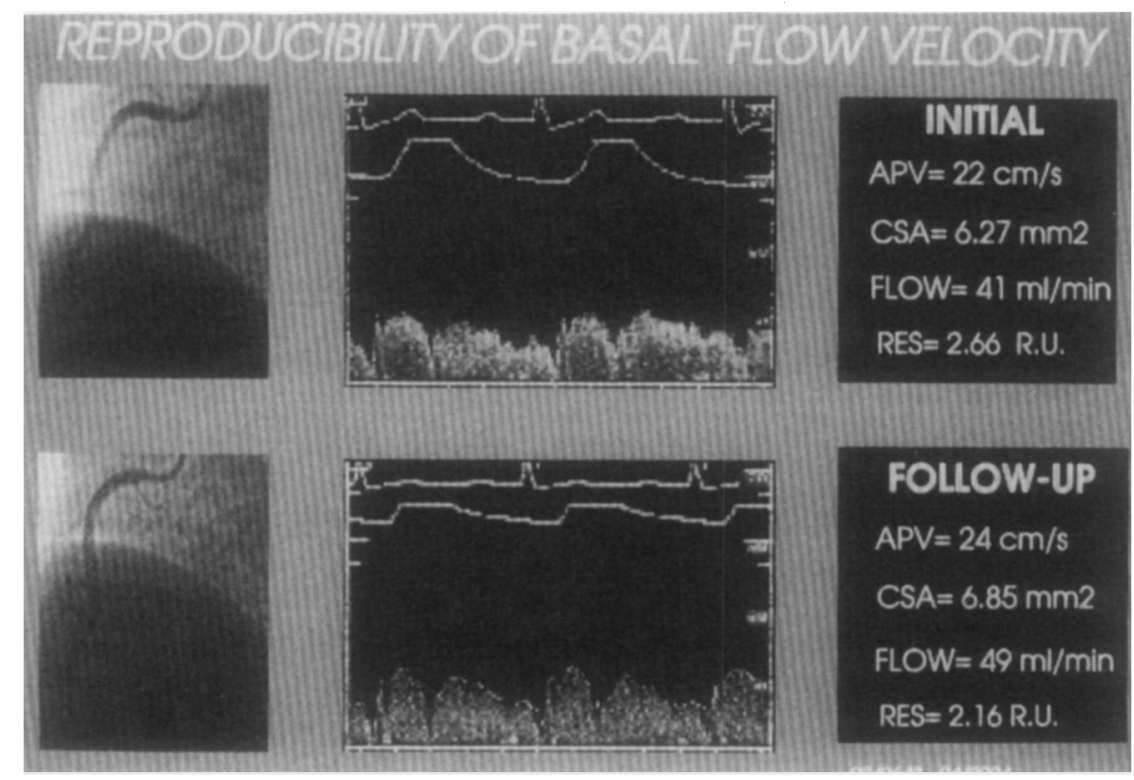

FIGURE 1. Doppler flow velocity measurements in the midsegment of an angiographically normal right coronary artery at baseline in the initial study lupper panels) and at 6-month follow-up (lower panels). Note the similar velocity pattern and the moderate changes in cross-sectional area (CSA), flow, and coronary flow resistance (RES). Velocity scale $=120$ $\mathrm{cm} / \mathrm{s} . \mathrm{APV}=$ time-averaged peak velocity; $\mathrm{RU}=$ resistance units $(\mathrm{mm} \mathrm{Hg} / \mathrm{ml} / \mathrm{min})$. 
for calibration. Cross-sectional area was calculated from the corresponding diameter assuming a circular arterial cross-section. Coronary flow ( $\mathrm{ml} / \mathrm{min})$ was calculated as the arterial cross-sectional area at the site of the Doppler sample volume $\left(\mathrm{mm}^{2}\right)$ multiplied by $1 / 2$ of the time-averaged peak velocity $(\mathrm{cm} / \mathrm{s})$ times 0.6 (correction factor); the coronary flow resistance $\left(\mathrm{mm} \mathrm{Hg} / \mathrm{min} / \mathrm{ml}^{-1}\right)$ was calculated as the mean aortic pressure $(\mathrm{mm} \mathrm{Hg})$ divided by the coronary flow $(\mathrm{ml} / \mathrm{min})$.

Matched measurements for initial and follow-up assessments were compared by using a two-tailed Student's $t$ test for paired data and linear regression analysis. Analysis of variance was used to test the correlation of hemodynamic and flow velocity changes. Mean difference and standard deviation of the signed difference were also calculated as proposed by Bland and Altman. ${ }^{5}$

Heart rate, mean aortic pressure, flow velocity, and coronary flow and flow resistance in baseline conditions and after maximal hyperemia are reported in Table I. The differences between initial and follow-up corresponding measurements of baseline velocity, hyperemic velocity, and coronary flow reserve, normalized for the mean of the two measurements, are displayed in Figure 2 (right panels). No significant differences between initial and follow-up measurements were present for baseline and hyperemic velocity and for coronary flow reserve. The standard deviation of the difference between initial and follow-up measurements was higher in baseline conditions $( \pm 31 \%)$ than during hyperemia $( \pm 23 \%)$. The largest dispersion of the data was observed for coronary flow reserve ( $\mathrm{SD}= \pm 36 \%$ ). Regression analysis (Figure 2, left panels) confirmed that a better correlation was present for the hyperemic measurements than for the baseline measurements ( $r=0.59$ and 0.46 , respectively). The correlation between initial and follow-up measurements was very poor for coronary flow reserve $(r=0.22)$. Absolute coronary flow and coronary flow resistance, on the contrary, showed a high correlation between corresponding measurements after a 6-month interval $(\mathrm{r}=0.65$ and 0.74 , respectively) (Figure 3). The changes in baseline velocity showed a significant positive correlation with the changes in heart rate $(r=0.39, p<0.05)$ (Figure 4, top panel). Since opposite changes were observed for the hyperemic velocity measurements (flow velocity reduction with increase in heart rate) (Figure 4, middle panel), coronary flow reserve showed a significant inverse correlation with heart rate (Figure 4, lower panel). No significant cor-

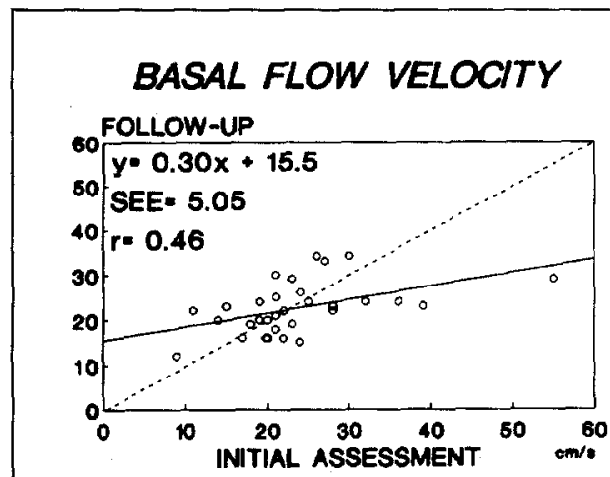

HYPEREMIC FLOW VELOCITY

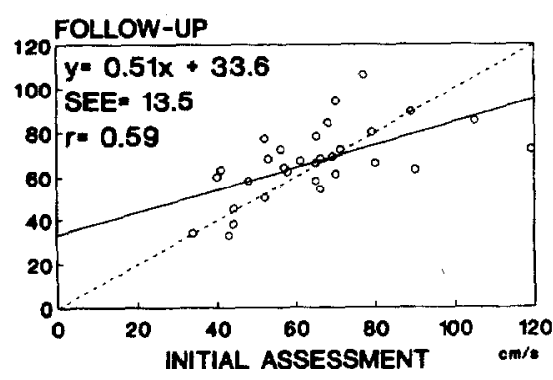

CORONARY FLOW RESERVE

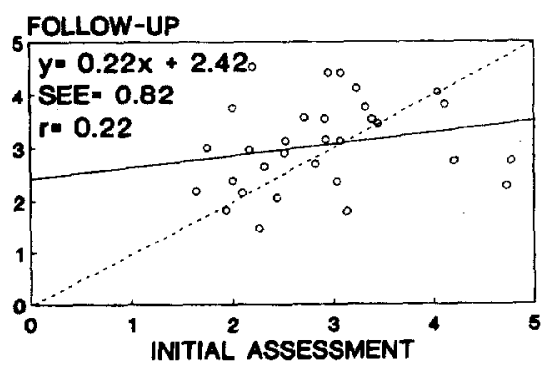

\section{BASAL FLOW VELOCITY}

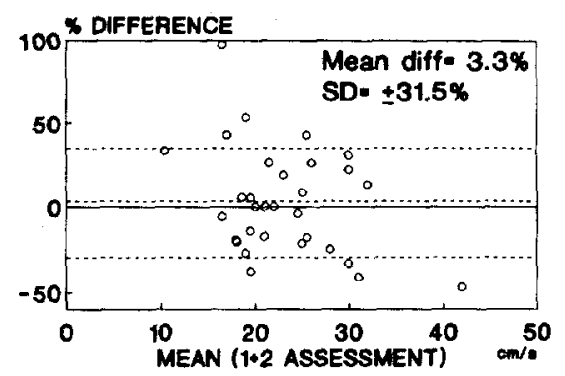

HYPEREMIC FLOW VELOCITY

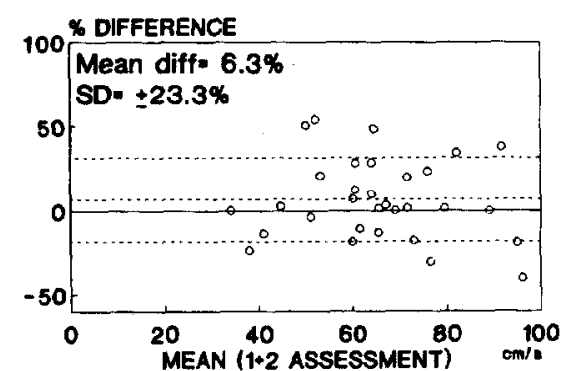

CORONARY FLOW RESERVE

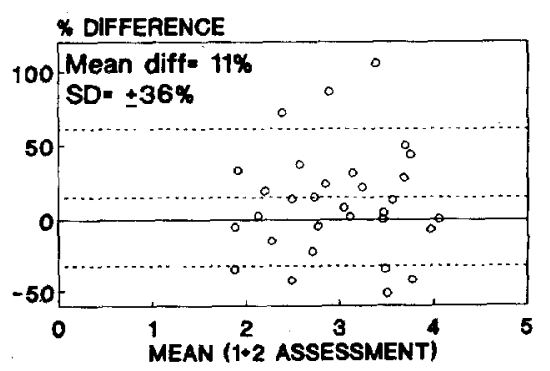

FIGURE 2. Left panels, comparison of follow-up and initial velocity measurements (fime-averaged peak velocity) using linear regression. The solid line represents the regression line, the dotted line represents the line of identity. Right panels, the difference between initial and follow-up velocity measurements, expressed as a percent of the initial velocity, is plotted against the mean of the 2 measurements. Nole that the lowest correlation and the highest standard deviation of the difference, indicating a large dispersion of the repeated measurements, were found for the measurements of coronary flow reserve (the ratio between mean hyperemic velocity and baseline velocity). 
relation was observed between pressure changes and flow velocity changes from baseline to follow-up.

Two factors may influence the reproducibility of velocity measurements over time: measurement variations due to technical limitations of the methods used for the assessment of velocity and area changes, and true biological differences that occur over the interval from the first to the second study. Minimizing the technical sources of measurement variability to be able to detect true changes due to pharmacological or mechanical interventions is a major challenge also for the most recent and sophisticated invasive methods of selective measurement of coronary velocity and diameter.

The large sample volume of the Doppler guide wire facilitates the acquisition of reproducible measurements, since moderate variations in the orientation of the Doppler crystal inside the vessel do not influence the final measurements. ${ }^{4}$ The recording of a high-quality Doppler spectrum, required in all cases, indicates that the centerline of flow is included in the Doppler sample volume. Since the Doppler shift is influenced by the cosine of the angle between the ultrasound beam and the maximal velocity vector, for a Doppler-tipped transducer, negligible changes are produced by relatively large deflections of the probe from the centerline of flow $(-6 \%$ for a $30^{\circ}$ angle). For a given flow, velocity is inversely correlated to the cross-sectional area at the site of the measurement. The better correlation found for flow measurements than that found for velocity measurements confirms the importance of the area changes determined by variations in coronary tone or by minor differences in the position of the guide wire along the vessel. It has been suggested that when intracoronary adenosine (12 to $18 \mu \mathrm{g})^{6,7}$ is used, a predilatation with nitrates is not strictly required, since this low dose has a selective effect on the resistance vessels without inducing changes in the area of the epicardial arteries. ${ }^{8}$ Intracoronary nitrates, however, remain highly recommended when reproducible measurements must be obtained in different phases of the same procedure (e.g., before and after balloon angioplasty) or in different procedures.

The basal coronary flow is ultimately determined by the myocardial oxygen demand. Heart rate, preload, mean aortic pressure, and inotropic status are the most important determinants of the cardiac workload and, consequently, of the myocardial oxygen consumption and baseline coronary flow. During maximal hyperemia, coronary flow is linearly, positively correlated with aortic pressure and negatively correlated with heart rate. This study shows that moderate spontaneous variations in heart rate over time are sufficient to impair the reproducibility of repeated measurements of baseline velocity and, to a lesser extent, of hyperemic velocity. Since opposite changes

\section{BASAL CORONARY FLOW $(\mathrm{m} / / \mathrm{min})$}

FOLLOW-UP

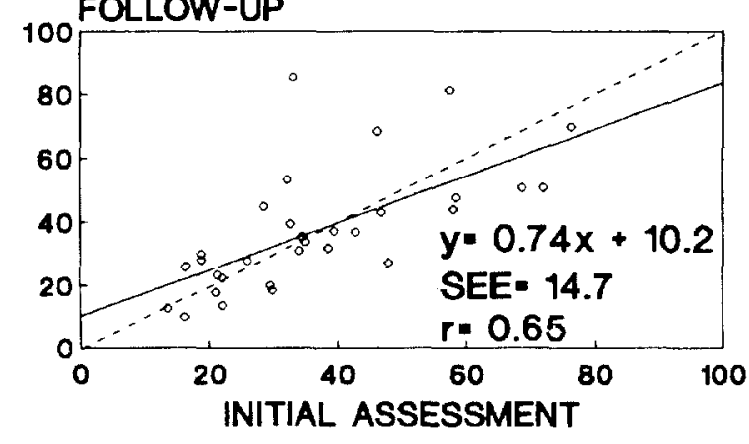

BASAL COR. RESISTANCE $(\mathrm{mmHg} / \mathrm{ml} / \mathrm{min}$ )

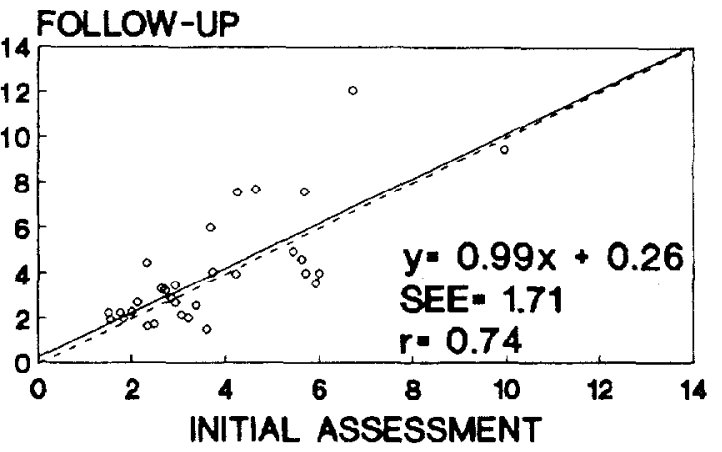

FIGURE 3. Regression analysis of coronary flow (left panel) and resistance (right panel) measurements in the initial and follow-up (4 to 7 months later) assessment. The solid line represents the regression line, the dotted line represents the identity line. Note the closer correlation of the measurements compared with the coronary flow velocity measurements (Figure 2). 


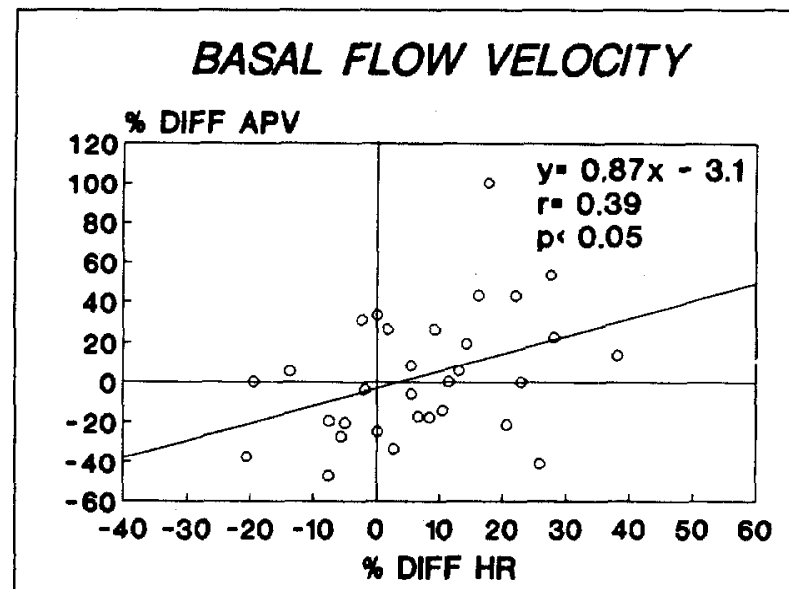

HYPEREMIC FLOW VELOCITY

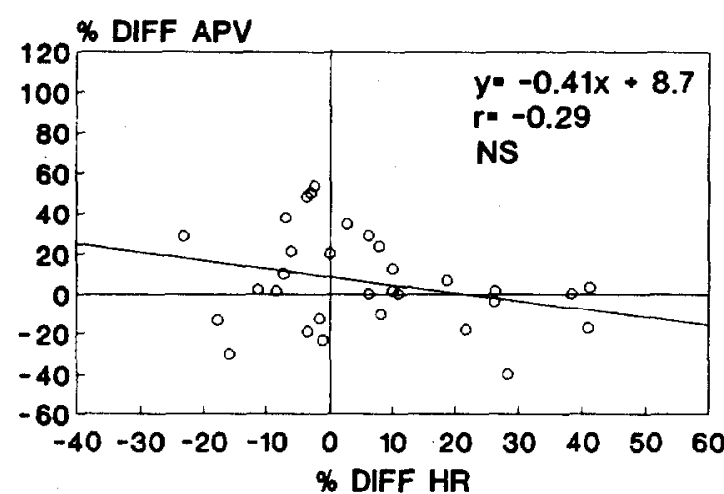

CORONARY FLOW RESERVE

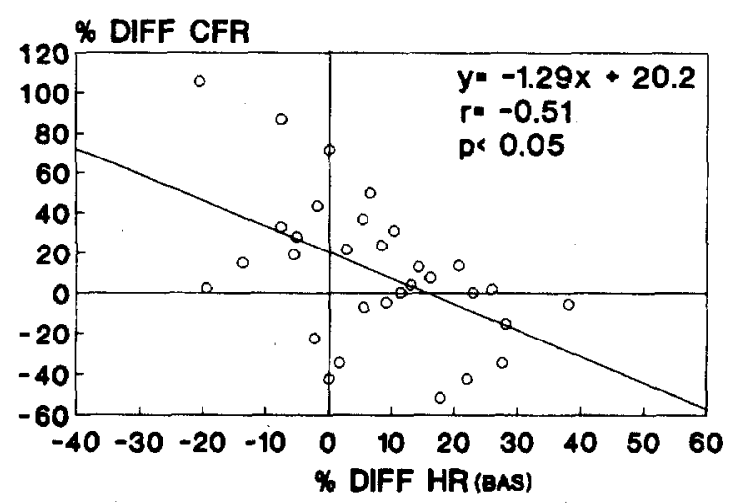

FIGURE 4. The percent difference in heart rate from the initial assessment is plotted against the percent difference in basal (top panel), hyperemic flow velocity (middle panel), and coronary flow reserve (lower panel) (in all cases follow-up lafter 4 to 7 months] minus initial assessment). Note the significant increase in basal flow velocity with the increase in heart rate and the opposite nonsignificant trend for hyperemic flow velocity. Consequently, a significant reduction of coronary flow reserve is observed with an increase in heart rate. APV = timeaveraged flow velocity; BAS = baseline; CFR = coronary flow reserve; $H R=$ heart rate. in baseline and hyperemic velocity occur with an increase in heart rate, as reported in previous studies ${ }^{9-11}$ coronary flow reserve decreased with an increase in heart rate. Atrial pacing or a normalization of the coronary flow reserve for the heart rate should be considered to overcome this limitation. The moderate changes in aortic pressure from the initial study to follow-up had a more limited influence on the changes in flow velocity and coronary flow reserve. When investigators are studying long-term changes, as in this study, the effects of riskfactor modifications in regard to smoking, elevated cholesterol levels, diabetes mellitus, and hypertension should be considered. These factors not only induce progression or regression of the atherosclerotic changes at an epicardial level, with consequent permanent changes in lumen area, but modify the endothelium-dependent and independent coronary vasodilatation of the resistance vessel. ${ }^{12}$

In conclusion, flow velocity measurements repeated after a 6-month interval show a variability, which is larger for baseline velocity and coronary flow reserve. This variability is correlated with the changes in heart rate and can be reduced by a normalization for the cross-sectional area at the site of the measurement (coronary flow) and for the aortic pressure at the time of the measurement (flow resistance).

1. Donohue TJ, Kern MJ, Aguirre FV, Bach RG, Wolford T, Bell CA. Assessing the hemodynamic significance of coronary artery stenoses: analysis of translesional pressure-flow velocity relations in patients. J Am Coll Cardiol 1993;22:449-458. 2. Serruys PW, Di Mario C, Meneveau N, de Jaegere P, Strikwerda S, de Feyter $\mathrm{PJ}$, Emanuelsson $\mathrm{H}$. Intracoronary pressure and tlow velocity from sensor tip guidewires. A new methodological comprehensive approach for the assessment of coronary hemodynamics before and after interventions. Am J Cardiol 1993;71: 41D-53D.

3. Anderson HV, Kirkeeide RL, Krishnaswami A, Weigelt LA, Revena M, Weisman HF, Willerson JT. Cyclic flow variations after coronary angioplasty in humans: clinical and angiographic characteristics and elimination with $7 \mathrm{E} 3$ monoclonal antiplatelet antibody. J Am Coll Cardiol 1994;23:1031-1037.

4. Doucette JW, Douglas Corl P, Payne HP, Flynn AE, Goto M, Nassi M, Segal J. Validation of a Doppler guide wire for intravascular measurement of coronary artery flow velocity. Circulation 1992;85:1899-1911.

5. Bland JM, Altman DG. Statistical methods for assessing agreement between two methods of clinical measurement. Lancet 1986;2:307-310.

6. Wilson RF, Wyche K, Christensen BV, Laxson DD. Effects of adenosine on human coronary arterial circulation. Circulation 1990;82:1595-1606.

7. Kern MJ, Deligonul U, Aguirre F, Hilton TC. Intravenous adenosine: continuous infusion and low dose bolus administration for determination of coronary vasodilator reserve in patients with and without coronary artery disease. J Am Coll Cardiol 1991;18:718-729.

8. Sudhir K, MacGregor JS, Barbant SD, Foster E, Filzgerald PJ, Chatterjee K, Yock PG. Assessment of coronary conductance and resistance vessel reactivity in response to nitroglycerin, ergonovine and adenosine: in vivo studies with simultaneous intravascular two-dimensional and Doppler ultrasound. J Am Coll Cardiol 1993;21:1261-1268.

9. Hongo M, Nakatsuka T, Watanabe N, Takenaka H, Tanaka M, Kinoshita $O$, Okubo S. Sekiguchi M. Effects of heart rate on phasic coronary blood flow pattern and flow reserve in patients with normal coronary arteries: a study with an intravascular Doppler catheter and spectral analysis. Am Heart J 1994;127:545-551. 10. McGinn $\mathrm{AL}$, White $\mathrm{CW}$, Wilson RF. Interstudy variability of coronary flow reserve: influence of heart rate, arterial pressure, and ventricular preload. Circulation 1990;81:1319-1330.

11. Rossen JD, Winniford MD. Effect of increases in heart rate and arterial pressure on coronary fluw reserve in humans. $J$ Am Coll Cardlol 1993;21:343-348.

12. Zeiher AM, Drexler $H$, Wollschlager $H$, Just $H$. Endothelial dysfunction of the coronary microvasculature is associated with impaired coronary blood flow regulation in patients with early atherosclerosis. Circulation 1991;84:1984-1992. 